\title{
Investigação sobre sincronização com homogeneidade entre vizinhos por meio da Transformada Wavelet Complexa Dual-Tree: resultados preliminares.
}

\author{
Maria Teodora Ferreira ${ }^{1}$ \\ Faculdade Bilac e Universidade do Vale do Paraíba - UNIVAP, Brasil \\ Celso B. N. Freitas ${ }^{2}$ \\ Programa de Pós-Graduação em Computação Aplicada (CAP) - INPE, Brasil \\ Margarete O. Domingues ${ }^{3}$ \\ Elbert E. N. Macau ${ }^{4}$ \\ Laboratório Associado de Computação e Matemática Aplicada - LAC, \\ Instituto Nacional de Pesquisas Espaciais - INPE, Brasil
}

\begin{abstract}
Resumo. O fato de conectar osciladores em grupos mais homogêneos ou heterogêneos modifica o comportamento do sistema como um todo? Trabalhos anteriores com o modelo de Kuramoto indicam que sim: redes com vizinhos mais similares entre si tendem a exibir maior sincronização para forças de acoplamento atrativas fracas. Apresentam-se aqui resultados preliminares com um modelo de atrator caótico não-coerente que também exibem essa propriedade. Esse cenário compreende mais um exemplo de aplicabilidade do método Discrete Complex Wavelet Approach - DCWA para atribuição de fase, propiciando o cálculo das métricas de sincronização necessárias.
\end{abstract}

Palavras-chave. Fase, Sincronização de fase, Transformada Wavelet Complexa Dual-tree, Redes de osciladores.

\section{Introdução}

Diversos estudos sobre sistemas multi-agentes, biológicos ou sociais, revelam que seus membros utilizam critérios de seleção não necessariamente aleatórios para o estabelecimento de vínculos. Há cenários nos quais indivíduos tendem a favorecer a heterogeneidade, como por exemplo, mulheres que não tomam pílula anticoncepcional rotularam como sexualmente mais agradável o odor de homens que apresentam maior disparidade genética em relação ao delas [10]. Por outro lado, adolescentes formam laços de amizade sem um critério absoluto de escolha, com a identidade étnica exercendo maior influência do que a orientação acadêmica [11].

\footnotetext{
${ }^{1}$ mteodoraf25@gmail.com

${ }^{2}$ cbnfreitas@gmail.com

${ }^{3}$ margarete.domingues@inpe.br

${ }^{4}$ elbert.macau@inpe.br
} 
Dentre questões subjacentes nesse contexto, pode-se perguntar se tais escolhas locais dos elementos influenciam o comportamento global do sistema. Inspirados por essas ideias, apresentam-se aqui avanços na pesquisa iniciada em [6] onde se estudou o impacto sobre a sincronização considerando redes de osciladores de fase não idênticos, sob diferentes padrões de conexão. Consideram-se configurações similares ou dissimilares as redes cujos elementos são mais homogêneos ou heterogêneos, respectivamente, em relação a seus vizinhos. Na literatura, tais fenômenos são também conhecidos como homofilia e heterofilia. Se não há clara tendência para nenhum desses extremos, dizemos que a configuração é neutra.

Para o modelo de Kuramoto com parâmetro de acoplamento fraco, foi detectado que configurações similares exibem maior sincronização do que configurações neutras e dissimilares. A fim de avaliar se este fenômeno também ocorre em cenários mais gerais, apresentam-se aqui experimentos numéricos preliminares considerando-se como elementos ativos da rede osciladores não-coerentes.

Contudo, as métricas utilizadas para quantificar a sincronização requerem a atribuição de uma variável de fase ao sinal, o que não é diretamente obtido pela série temporal de tais osciladores sem centro de rotação bem definido.

Várias técnicas vêm sendo desenvolvidas para a obtenção da fase. Em [4] propõe-se um método, chamado Discrete Complex Wavelet Approach (DCWA), baseado em uma transformada wavelet, a Transformada Wavelet Complexa Dual-Tree (DT-CWT), para obter a fase de sistemas caóticos e de conjunto de dados experimentais, a fim de analisar o fenômeno de sincronização de fase. A aplicabilidade do método DCWA já foi verificada em sistemas caóticos de Rössler em regime de fase coerente e não coerente [2,3], em sistemas caóticos de Lorenz [1] e no Modelo de Kuramoto com diferentes configurações [4,5].

Como teste de conceito, redes de osciladores Rössler não-coerentes sob acoplamento fraco são consideradas com configurações similares, neutras e dissimilares. Após a integração numérica de tais sistemas, um par de osciladores adjacentes é escolhido aleatoriamente e aplica-se a DCWA para atribuição de fase. A fim de comparar os resultados obtidos da DCWA, também aplica-se a transformada de Hilbert para calcular a fase. Com isso, calcula-se o índice de sincronização parcial [7] com o qual pode-se comparar a emergência da sincronização de fase resultante em cada configuração de vizinhança.

A relevância deste estudo compreende a generalização de parte dos resultados obtidos em [6], apontando que, mesmo para tipos de osciladores mais sofisticados, o grau de similaridade entre o membros e seus vizinhos na rede exerce notável impacto nas características de todo o conjunto. O entendimento e a mimetização desses fenômenos podem ser úteis para uma ampla gama de aplicações envolvendo sincronização em redes, tais como malhas de produção e consumo de energia, propagação de solidariedade em comunidades humanas e o movimento coletivo de veículos autônomos [6]. Mais ainda, a demonstração da aplicabilidade da DCWA [4] para atribuição de fase em mais um contexto também é uma contribuição do presente trabalho. 


\section{Metodologia para atribuição de fase}

Nesta seção apresenta-se o método DCWA para a atribuição de fase. O cálculo da fase via transformada de Hilbert também é descrito.

\section{$2.1 \quad$ DCWA}

Em [4] propõe-se um método, chamado Discrete Complex Wavelet Approach (DCWA), o qual baseia-se no uso da Transformada Wavelet Complexa Dual-Tree (do inglês, DualTree Complex Wavelet Transform - DT-CWT). Na prática, a DT-CWT emprega duas Transformada Wavelet Discreta reais. Para informações veja $[8,9,12]$.

O método DCWA consiste em obter a fase a partir dos coeficientes wavelet complexo obtidos pela decomposição da série temporal utilizando a DT-CWT. Assim, a série temporal $x$ é analisada em $j$ níveis de decomposição, com $j=1, \ldots, \mathcal{L}$. Em cada nível de decomposição $j$, o coeficiente wavelet complexo $\boldsymbol{d}^{j}$ é obtido por meio da análise DT-CWT. Como a DT-CWT é uma transformada decimada, na escala $j$ a dimensão do vetor contendo os coeficientes $\boldsymbol{d}^{j}$ é igual a $N^{j}=2^{L-j}$, em que $N^{j}$ denota o número de pontos $N$ na escala $j$. Em seguida, a energia $E^{j}$ para cada escala $j$ é calculada como o módulo ao quadrado do coeficiente wavelet complexo $\boldsymbol{d}^{j}$ por meio da equação $E^{j}(n)=\left|\boldsymbol{d}^{j}(n)\right|^{2}$. O espectro de energia global $\mathbb{E}^{j}$ é calculado por meio da equação $\mathbb{E}^{j}=\sum_{n} E^{j}(n)$. Posteriormente, procura-se a escala $J$ a qual apresenta a energia global máxima do sistema utilizando-se a condição $\mathbb{E}^{J}=\max _{j} \mathbb{E}^{j}$. Depois de encontrada a escala $J$, a fase é calculada nesta escala $J$, denotada por $\phi^{J}$ e dada por $\phi^{J}(t)=\operatorname{atan} 2\left(\underline{d}^{J}, \bar{d}^{J}\right)$. Aqui, $\underline{d}^{J}$ é a parte imaginária do coeficiente wavelet complexo na escala $J, \bar{d}^{J}$ é a parte real do coeficiente wavelet complexo na escala $J$ e a função atan2 é a função arco-tangente de dois argumentos.

\subsection{Transformada de Hilbert}

A Transformada de Hilbert (TH) de uma dada série temporal $f(t)$ é definida por $\mathcal{H}(t)=\frac{1}{\pi} \int_{-\infty}^{\infty} \frac{f(\tau)}{t-\tau} d \tau$. A construção do sinal analítico complexo $\zeta(t)$ a partir da série temporal $f(t)$ utilizando a TH pode ser feita por meio da equação $\zeta(t)=f(t)+\imath f_{\mathcal{H}}(t)=$ $A(t) e^{\imath \phi_{\mathcal{H}}(t)}$, em que $f_{\mathcal{H}}(t)$ é a transformada de Hilbert de $f(t)$. Essa equação fornece a fase $\phi_{\mathcal{H}}(t)$ e a amplitude instantânea $A(t)$.

\section{Aplicação}

Nesta seção, o método DCWA será aplicado para a obtenção da fase e, posteriormente, na análise de sincronização em redes de osciladores não idênticos acoplados sob configurações similares, neutra e dissimilares introduzidas em [6].

Considere-se $\mathcal{A}$ um grafo simples e conectado com $N$ vértices e $E$ arestas. Além disso, $\omega_{1}, \ldots, \omega_{n} \in \mathbb{R}$ parametrizam a dinâmica desacoplada de cada um dos $N$ osciladores, ou seja, desconsiderando-se qualquer influência de outros osciladores. Ao permitir somente 
a permutação dos $N$ osciladores, caracterizados por seus parâmetros $\omega_{i}$, dentre os $N$ vértices do grafo $\mathcal{A}$, chamamos de configurações similares ou dissimilares as permutações que favorecem vizinhos homogêneos ou heterogêneos, respectivamente. Por outro lado, na ausência de forte tendência homogênea ou heterogênea, dize-se que há uma configuração neutra. A definição matemática e os algoritmos para obtenção de tais configurações podem ser encontrados em [6]. Para o escopo deste trabalho, ilustra-se tais conceitos com redes do tipo Watts-Strogatz com $N=100$ osciladores e $E=200$ arestas, conforme mostrado na Figura 1. Nesse exemplo $\omega_{1}, \ldots, \omega_{N}$ foram escolhidos aleatoriamente com distribuição uniforme entre 0.9 e 1.1 .

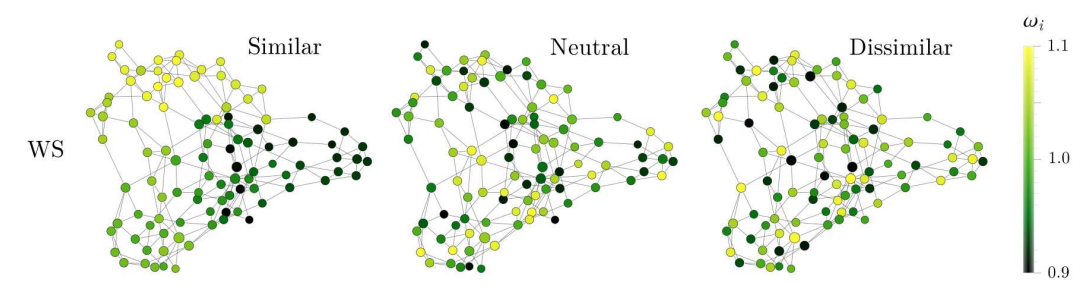

Figura 1: Redes similares, neutras e dissimilares estudadas neste artigo.

Ainda em [6], para valores baixos de acoplamentos em um modelo de Kuramoto com osciladores não-idênticos, foram apresentadas evidências numéricas de que redes similares exibem maior sincronização do que redes neutras e dissimilares. Neste trabalho, apresentam-se resultados preliminares por meio do método DCWA de que o mesmo fenômeno ocorre para outros tipos mais gerais de osciladores, a saber, osciladores de Rössler não-idênticos com atrator não-coerente.

A dinâmica que rege cada oscilador $i=1, \ldots, N$ é definida por [2]:

$$
\begin{aligned}
\dot{x}_{i} & =-\omega_{i} y_{i}-z_{i} \\
\dot{y}_{i} & =\omega_{i} x_{i}+a y_{i}+\eta \sum_{j \in \mathcal{N}_{i}}\left(y_{j}-y_{i}\right) \\
\dot{z}_{i} & =b+z_{i}\left(x_{i}-c\right),
\end{aligned}
$$

em que $\eta \in \mathbb{R}$ é o parâmetro de acoplamento do sistema; $\mathcal{N}_{i}$ representa o conjunto dos nós adjacentes ao vértice $i$. Utilizam-se $a=0.2925, b=0.4, c=-8.5$, a fim de obterse atratores Rössler não-coerentes, ou seja, sem um centro de rotação bem definido. A caracterização das dinâmicas não-idênticas na redes são representadas pelo parâmetro $\omega_{i}$. A Fig. 2 exibe trajetórias dos osciladores desacoplados $(\eta=0)$ para diferentes valores de $\omega_{i}$.

Escolhe-se heuristicamente um valor baixo de acoplamento $(\eta=0.05)$ e efetua-se a integração numérica do sistema 1 a partir de condições iniciais aleatórias próximas à região do atrator caótico. Esse procedimento foi repetido para cada uma das três configurações de vizinhança. A partir daí atribuí-se variáveis de fase via DCWA utilizando-se a coordenada $x$ para dois dos osciladores na rede, $i=1,2$. Com isso, pode-se computar o chamado 

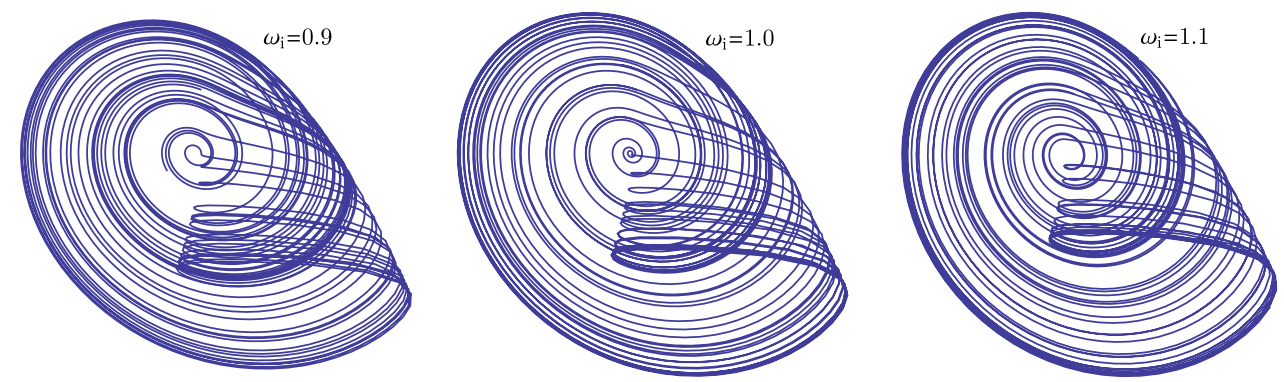

Figura 2: Projeção no plano $x, y$ dos osciladores de Rössler não-coerentes isolados, com diferentes valores do parâmetro $\omega_{i}$.

índice de sincronização parcial [7] entre esse par de osciladores

$$
S=S_{1,2}=S_{2,1}:=\left|\lim _{\Delta t \rightarrow \infty} \frac{1}{\Delta t} \int_{t_{r}}^{t_{r}+\Delta t} \mathrm{e}^{\mathrm{i}\left(\theta_{1}(t)-\theta_{2}(t)\right)} \mathrm{d} t\right|,
$$

em que $t_{r}$ é o tempo de transiente suprimido, no nosso caso, utilizou-se 100u.t. Os osciladores 1,2 entram em travamento de fase, isto é, $\theta_{i}(t)-\theta_{j}(t)$ converge para uma constante, se e somente se, $S_{i j}=1$. A medida que esse índice decresce, formas mais fracas de sincronização começam a aparecer. Finalmente, $S_{i j} \approx 0$ indica ausência de sincronização, quando as trajetórias dos osciladores estão muito pouco relacionadas. Em suma, pode-se interpretar que quanto mais próximo $S$ está de 1 , maior a sincronização entre o par de osciladores.

A fim de comparar os resultados obtidos do índice de sincronização via atribuição de fase usando a DCWA, utilizou-se também a transformada de Hilbert para a atribuição de fase. A Tabela 1 apresenta os índices de sincronização computados entre o par de osciladores 1,2 para a configuração similar, neutra e dissimilar considerando a utilização da DCWA e da Transformada de Hilbert para atribuição de fase.

Tabela 1: Índices de sincronização computados entre o par de osciladores 1,2 para a configuração similar, neutra e dissimilar considerando a utilização da DCWA e da transformada de Hilbert na atribuição de fase.

\begin{tabular}{|c|c|c|}
\hline Configuração & DCWA & Hilbert \\
\hline similar & $S=0.404$ & $S=0.343$ \\
\hline neutra & $S=0.216$ & $S=0.191$ \\
\hline dissimilar & $S=0.181$ & $S=0.156$ \\
\hline
\end{tabular}

Portanto, também nesta classe de osciladores, observa-se que a emergência da sincronização ocorre de maneira mais proeminente para redes similares, considerando-se valores 
fracos de acoplamento. Nota-se que na Tabela 1 os valores dos índices de sincronização são semelhantes entre as duas técnicas utilizadas, sendo os valores da DCWA sempre ligeiramente superiores.

\section{Conclusão}

Neste trabalho, avalia-se que a técnica Discrete Complex Wavelet Approach (DCWA) é eficiente para o cálculo das fases em rede de osciladores de Rössler não idênticos e nãocoerentes. A partir daí, pode-se computar o índice de sincronização entre dois nós da rede e observar que configurações similares exibem níveis mais altos de sincronização em relação a redes neutras e dissimilares. Por ser uma técnica mais simples de aplicação e ter características multiescala pretende-se futuramente estender esse estudo para estudar a evolução da sincronização considerando-se todos os nós da rede, outros valores de acoplamento, bem como outros tipos de grafos de acoplamento.

\section{Agradecimento}

À CAPES e ao CNPq (processos números 309667/2013-5, 306828/2010-3, 306038/20153, 483226/2011-4, 10571/13-2, 304582/2010-7) e FAPESP (2011/50151-0) pelo suporte financeiro a este trabalho.

Ao prof. Nick Kingsbury pelo algoritmo da DT-CWT e por ter apresentado dois minicursos sobre este assunto no WWlet/CNMAC 2012.

\section{Referências}

[1] M. T. Ferreira, R. Follmann, M. O. Domingues, and E. E. N. Macau. Aplicação da transformada wavelet complexa dual-tree na detecção de sincronização de fase entre sistemas caóticos de lorenz. In Anais do Congresso de Matemática Aplicada e Computacional - CMAC Sudeste, Bauru, São Paulo, Brasil, 2013.

[2] M. T. Ferreira, R. Follmann, M. O. Domingues, and E. E. N. Macau. Detecting phase synchronization in coupled chaotic noncoherent oscillators by using complex wavelet transform. In 11th International Conference on Vibration Problems - ICOVP, Lisbon, 2013.

[3] M. T. Ferreira, R. Follmann, M. O. Domingues, and E. E. N. Macau. Transformada wavelet complexa dual-tree e a detecção da sincronização de fase entre sistemas com dinâmica caótica. In 10th Conferência Brasileira de Dinâmica, Controle e Aplicações - DINCON, Águas de Lindóia, São Paulo, Brasil, 2011. DOI: 10.5540/DINCON.2011.001.1.0184.

[4] M. T. Ferreira, C. B. N. Freitas, M. O. Domingues, and E. E. N. Macau. The discrete complex wavelet approach to phase assignment and a new test bed for related methods. Chaos, 25(1), 2015. DOI: 10.1063/1.4906814. 
[5] M. T. Ferreira, C. B. N. Freitas, M. O. Domingues, and E. E. N. Macau. Modelo de kuramoto e a verificação da diferença de fase usando uma metodologia baseada na transformada wavelet complexa dual-tree: resultados preliminares. In Proceeding Series of the Brazilian Society of Applied and Computational Mathematics, 2013.

[6] C. Freitas, E. Macau, and R. L. Viana. Synchronization versus neighborhood similarity in complex networks of nonidentical oscillators. Phys. Rev. E, 92(3):032901, 2015. DOI: 10.1103/PhysRevE.92.032901.

[7] J. Gómez-Gardeñes, Y. Moreno, and A. Arenas. Paths to synchronization on complex networks. Phys. Rev. Lett., 98:034101, 2007. DOI: 10.1103/PhysRevLett.98.034101.

[8] N. Kingsbury. Complex wavelets for shift invariant analysis and filtering of signals. Appl. Comput. Harmon. Anal., 10(3):234-253, 2001. DOI: 10.1006/acha.2000.0343.

[9] N. Kingsbury. Image processing with complex wavelets. Phil. Trans. Royal Society A, 357(1760):2543-2560, 1999. DOI: 10.1098/rsta.1999.0447.

[10] P. S. C. Santos, J. A. Schinemann, J. Gabardo, and M. G. Bicalho. New evidence that the mhe influences odor perception in humans: a study with 58 southern brazilian students. Horm. Behav., 47(4):384-388, 2005. DOI: 10.1016/j.yhbeh.2004.11.005.

[11] D. Schultz and S. E. Schultz. Theories of personality. Cengage Learning, USA, eleventh edition edition, 2012.

[12] I. W. Selesnick, R. G. Baraniuk, and N. G. Kingsbury. The dual-tree complex wavelet transform. IEEE Signal Proc. Mag., 22(6):123-151, 2005. DOI: 10.1109/MSP.2005.1550194. 